\title{
A relação entre a legislação brasileira sobre a obrigatoriedade de dispositivos de retenção para crianças de zero a quatro anos ocupantes de veículos e os números de feridos e mortos no trânsito
}

\author{
The relationship between Brazilian legislation on the mandatory \\ use of restraint devices for zero- to four-year-old children \\ in vehicles and the numbers of injured and dead in traffic accidents
}

Júlio César Matos de Oliveira (https://orcid.org/0000-0002-5139-173X) ${ }^{1}$

Luiz Honorato da Silva Júnior (https://orcid.org/0000-0002-2840-3579) ${ }^{1}$

Alexandre Nascimento de Almeida (https://orcid.org/0000-0002-9113-0729) ${ }^{1}$

${ }^{1}$ Programa de Pós-

Graduação em Gestão

Pública, Universidade de

Brasília. Vila Nossa Senhora

de Fátima, - Planaltina.

73345-010. Brasília

DF Brasil.

julio.jcmo@yahoo.com.br

\begin{abstract}
The scope of this article is to address the relationship between mandatory restraint devices and hospitalizations and deaths of zero- to fou$r$-year-old age children in traffic accidents in Brazil. Based on the mandatory use of child restraint devices for children up to 4 years of age under Brazilian traffic legislation in 2010, the authors apply data from the DataSUS and Denatran databases to analyze the time series of hospitalizations and deaths of zero- to four-year-old age children in traffic accidents between September 2005 and August 2015. Two cut-off sample periods were examined, the first consisting of an analysis from 2005 to 2015, which was subsequently subdivided into two samples, namely before and after the requirement. The results of both cut-off sample periods suggest that demands concerning the use of restraint devices led to decreased hospitalizations and deaths of children due to traffic accidents in the zero- to four-year-old age group, with a prevalence of decreased rates of hospitalization over deaths.
\end{abstract}

Key words Traffic accidents, Children, Restraint devices
Resumo Este artigo aborda a relação entre a obrigatoriedade de dispositivos de retenção (cadeirinhas) e as internações e mortes de crianças de zero a quatro anos em sinistros de trânsito no Brasil. A partir da obrigatoriedade do uso de dispositivos de retenção para crianças de até quatro anos pela legislação de trânsito brasileira, ocorrida no ano de 2010, os autores utilizam os dados do sistema DataSUS e do Denatran para analisar as séries temporais das internações e mortes de crianças de zero a quatro anos em sinistros de trânsito ocorridas no período de setembro de 2005 a agosto de 2015. São realizados dois recortes. Primeiro, uma análise do período compreendido entre os anos de 2005 e 2015, posteriormente esse período foi dividido em duas amostras, antes e depois da obrigatoriedade. Os resultados em ambos os recortes sugerem que a exigência da utilização dos dispositivos de retenção gerou redução de internações e mortes de crianças por sinistros de trânsito na faixa etária de zero a quatro anos, com prevalência da redução de internações sobre a de mortes.

Palavras-chave Sinistros de trânsito, Crianças, Dispositivos de retenção 


\section{Introdução}

Os sinistros de trânsito impactam toda a sociedade, trazendo prejuízos significativos para o país ${ }^{1,2}$ De forma geral, estudos demonstram que o Código de Trânsito Brasileiro (CTB), Lei no 9.503/97, teve papel importante na redução dos sinistros de trânsito desde a sua publicação, mas sua capacidade de transformar a realidade da violência no trânsito se mostrou limitada, principalmente pela timidez das políticas públicas desenvolvidas ao longo do tempo e a quantidade reduzida de ações de fiscalização $0^{3-6}$.

Diante desse cenário, medidas para reduzir o número de sinistros e sua gravidade são periodicamente adotadas por todos os países, inclusive o Brasil. A OMS destaca que os seis principais fatores de risco em sinistros de trânsito são: excesso de velocidade, condução sob efeito de álcool, condução sob efeito de drogas, não uso de capacetes pelos motociclistas, não uso de cintos de segurança e sistemas de retenção de crianças ${ }^{1}$.

A OMS aponta que a utilização de dispositivos de retenção reduz em cerca de $60 \%$ a probabilidade de morte de crianças em sinistros de trânsito, com incremento nos casos de crianças de até quatro anos de idade. De 179 países, apenas 33 possuem, simultaneamente, normas sobre sistemas de retenção para crianças com base na idade, altura ou peso e normas que restringem por idade ou altura as crianças que podem se sentar no banco da frente dos veículos. Esses países que adotam as melhores práticas em relação ao transporte de crianças em veículos automotores representam somente $9 \%$ da população mundial ${ }^{1}$.

A Academia Americana de Pediatria recomenda que os dispositivos de retenção sejam voltados para a retaguarda o maior tempo possível, que sejam voltados para frente apenas para crianças com pelo menos quatro anos de idade, que os assentos elevatórios sejam utilizados apenas em crianças com pelo menos oito anos de idade, que os cintos de segurança utilizados com assentos elevatórios sejam de três pontas, ou seja, que protejam colo e ombros, e que todas as crianças menores de 13 anos andem nos bancos traseiros dos veículos?

A relevância da adoção dos dispositivos de retenção para a redução de lesões e mortes de crianças em sinistros de trânsito é indicada na literatura internacional desde a década de $1970^{8}$. Desde então, diversos estudos apontam os dispositivos de retenção como medidas eficientes para a proteção de crianças no trânsito, sobretudo as menores quatro anos, e sua maior eficácia em relação aos cintos de seguranç̧̧ ${ }^{9-17}$.

No Brasil, o CTB estabeleceu que as crianças com idade inferior a dez anos devem ser transportadas nos bancos traseiros, salvo exceções regulamentadas pelo Conselho Nacional de Trânsito $(\text { CONTRAN })^{18}$. No ano de 2008, o CONTRAN editou a Resolução 277/2008, que definiu o que são os dispositivos de retenção e estabeleceu sua obrigatoriedade para crianças entre zero e sete anos e meio ${ }^{19}$. Para a norma, o dispositivo de retenção é:

Dispositivo de retenção para crianças é o conjunto de elementos que contém uma combinação de tiras com fechos de travamento, dispositivo de ajuste, partes de fixação e, em certos casos, dispositivos como: um berço portátil porta-bebê, uma cadeirinha auxiliar ou uma proteção antichoque que devem ser fixados ao veículo, mediante a utilização dos cintos de segurança ou outro equipamento apropriado instalado pelo fabricante do veículo com tal finalidade.

A partir da própria definição de dispositivo de retenção apresentada pela Resolução 277/2008, conclui-se que o "assento de elevação" não é um dispositivo de retenção tal como definido pela norma, mas um suporte para que a criança possa utilizar adequadamente o cinto de segurança do próprio veículo.

É importante destacar que a Resolução 277/2008 apresentou exceções à obrigatoriedade da utilização dos dispositivos de retenção, deixando de fora das exigências os veículos de transporte coletivo, os de aluguel, os de transporte autônomo de passageiro (táxis), os veículos escolares e os demais com peso bruto total superior a 3,5t. Assim, a obrigatoriedade se restringiu a automóveis, caminhonetes e caminhonetas, o que representava, segundo dados do Departamento Nacional de Trânsito (DENATRAN), 65,80\% da frota brasileira de veículos automotores em $2013^{20}$.

No Brasil, efeito do uso de dispositivos de retenção na redução de lesões e mortes de crianças em sinistros de trânsito ainda não foi devidamente explorado, no entanto as pesquisas já realizadas têm indicado a efetividade desses equipamentos $^{21-24}$.

Este artigo analisa a relação entre a obrigatoriedade do uso de dispositivos de retenção em veículos automotores e o número de internações e mortes de crianças de zero a quatro anos no trânsito. 


\section{Métodos}

O estudo realizado se trata de uma pesquisa descritiva ecológica, em que utilizamos a análise de séries temporais. Para a realização do estudo, adotamos que o conceito de dispositivos de retenção se enquadra em equipamentos chamados no Brasil de "bebê conforto" e "cadeirinha", utilizados em crianças de até um ano, no primeiro caso, e de um a quatro anos no segundo caso. A decisão metodológica se deu pelo fato de que os assentos de elevação, usados para crianças de quatro a sete anos e meio, não são dispositivos de retenção, mas um equipamento utilizado para que a criança se ajuste ao próprio cinto de segurança do veículo.

Extraímos os dados a respeito das internações e mortes de crianças de zero a quatro anos em sinistros de trânsito no período de 2005 a 2015 do banco de dado do Sistema de Informações Hospitalares $^{25}$, para as internações, e do Sistema de Informações sobre Morbidades ${ }^{26}$, para as mortes. Selecionamos os seguintes dados para as internações: por local da internação; região segundo ano/mês da internação; Grande Grupo CID 10: V01-V99 sinistros de transporte; Grupo CID 10: ocupante de automóvel traumatizado em acidente de transporte, ocupante de caminhonete traumatizado em acidente de transporte; Categoria CID 10: V40-V59; faixa etária: menor de um ano, um a quatro anos; período 2005 a 2015. Já para os óbitos, selecionamos: óbitos por ocorrência por região segundo ano/mês do óbito; Grande Grupo CID 10: V01-V99 sinistros de transporte; Grupo CID 10: ocupante de automóvel traumatizado em acidente de transporte, ocupante de caminhonete traumatizado em acidente de transporte; Categoria CID 10: V40-V59; faixa etária: menor de um ano, um a quatro anos; período de 2005 a 2015.

Utilizamos o banco de dados do DENATRAN para o levantamento da evolução mensal da frota de automóveis, caminhonetes e caminhonetas no Brasil entre os anos de 2005 e 2015.

A fim de que a variação da frota de veículos não influenciasse nos resultados da pesquisa, empregamos as séries temporais das internações e mortes a partir do indicador "mortes por milhão de veículos”. Mello Jorge ${ }^{27}$ demonstra que, entre 1996 e 2011, a frota brasileira cresceu quase $155 \%$. No mesmo sentido, a taxa de motorização, que é a relação entre o número de veículos e a população, subiu de 170,8 veículos para cada 1.000 habitantes em 1995 para 365,6 em 2011, um aumento de $114 \%$.

Em regra, a utilização dos dispositivos de retenção é obrigatória somente para automóveis, caminhonetes e caminhonetas, assim, a fim de não incluir na pesquisa veículos que não são obrigados a transportar crianças nesses dispositivos, na extração dos dados sobre internações, mortes e frota de veículos, utilizamos apenas informações relativas a automóveis, caminhonetes e caminhonetas. Os dados sobre a evolução da frota de automóveis, caminhonetes e caminhonetas coletados no presente trabalho demonstram que, entre setembro de 2005 e agosto de 2015, houve um crescimento de $95 \%$, passando de 29.972.809 veículos, para 58.561.004 veículos ${ }^{20}$.

Selecionamos os períodos adotados pela pesquisa para a extração dos dados a partir das peculiaridades da implementação da Resolução 277/2008 do CONTRAN. A resolução foi publicada em 9 de setembro de 2008, no entanto, após um período destinado a campanhas educativas, a obrigatoriedade de utilização dos dispositivos de retenção foi iniciada em $1^{\circ}$ de setembro de $2010^{28}$.

A partir do exposto acima, definimos um período de 60 meses, entre setembro de 2005 e agosto de 2010, para a análise dos dados referentes ao período anterior à obrigatoriedade de utilização dos dispositivos de retenção, e um período de 60 meses, entre setembro de 2010 e agosto de 2015, para a análise dos dados referentes ao período posterior à obrigatoriedade de utilização dos mesmos. A limitação do SIH/DATASUS e do SIM/DATASUS para a disponibilização dos dados de internações e mortes influenciou na formação da série temporal utilizada na pesquisa.

Realizamos o ajuste da série temporal a partir do método de Box e Jenkins ${ }^{29}$, com a aplicação de um modelo autorregressivo integrado de médias móveis (ARIMA). A estacionariedade das séries foi verificada a partir dos testes de raiz unitária ADF e DFGLS. A sazonalidade das séries foi testada por meio do correlograma, e a dessazonalização das séries realizadas pelo método X12-ARIMA. Por fim, os resíduos foram verificados com a aplicação dos testes de White (heterocedasticidade), de normalidade e de correlação.

A fim de verificar a influência da obrigatoriedade dos dispositivos de retenção, utilizamos a técnica de análise de intervenção. Morettin e Toloi ${ }^{30}$ esclarecem que a análise de intervenção tem por objetivo avaliar o impacto de determinado evento no comportamento de uma série temporal. Para isso, criamos uma variável dummy, binária, com valor 0 para o período entre setembro de 2005 e agosto de 2010, e valor 1 para o período entre setembro de 2010 e agosto de 2015 .

Com o intuito de deixar o estudo mais robusto, as séries temporais das internações e das 
mortes foram seccionadas em dois períodos cada uma, com o objetivo de analisar a existência de alteração de intercepto e de tendência para as séries antes e depois da obrigatoriedade da utilização dos dispositivos de retenção. Assim, o mesmo fluxo procedimental realizado com as séries completas foi executado nas séries seccionadas (setembro de 2005 a agosto de 2010 e setembro de 2010 a agosto de 2015).

\section{Resultados}

Entre os anos de 2005 e 2015 houve, no Brasil, 3.008 internações e 1.730 mortes de crianças de zero a quatro anos em decorrência de sinistros de trânsito envolvendo automóveis, caminhonetas e caminhonetes. O Gráfico 1 apresenta a distribuição da média mensal de internações e mortes no período analisado pela pesquisa, além de sua tendência linear.

A análise do Gráfico 1 sugere uma tendência de queda no número de mortes e, principalmente, no número de internações após a obrigatoriedade dos dispositivos de retenção para crianças de zero a quatro anos no Brasil.

Após a obtenção dos resultados descritivos, apresentados no Gráfico 1, procedemos com os testes para a realização dos estudos inferenciais, a partir da aplicação do método de análise de séries temporais. A análise da série temporal das internações identificou uma sazonalidade de 12 meses na série, o que exigiu a sua dessazonalização. Confirmamos a estacionariedade da série pelos testes $\mathrm{ADF}$, ao nível de $1 \%(\mathrm{n}=-7.617$, $\mathrm{p}=0.000)$, e DFGLS, ao nível de $1 \%(\mathrm{n}=-7.625$, $\mathrm{p}=0.000)$. Assim, por meio do correlograma e do teste de raiz unitária, especificamos um modelo ARIMA $(1,0,0)$ para a série internações. Confirmamos a homogeneidade do modelo proposto pelo teste de White $(\mathrm{p}=0.000)$ e o teste de normalidade indicou a probabilidade de $6 \%$ e rejeitou a presença de correlação serial.

$\mathrm{Na}$ análise da série de mortes, também identificamos uma sazonalidade de 12 meses. Após a dessazonalização, a estacionariedade foi confirmada pelos testes ADF, ao nível de $1 \%$ ( $\mathrm{n}=$-9.776, $\mathrm{p}=0.000)$, e DFGLS, ao nível de $1 \%$ $(\mathrm{n}=-9.250, \mathrm{p}=0.000)$. Aplicamos um modelo ARIMA $(4,0,3)$ para ajuste da série. Confirmamos a homogeneidade do modelo proposto pelo teste de White $(\mathrm{p}=0.016)$ e o teste de normalidade indicou a probabilidade de $90 \%$ e rejeitou a presença de correlação serial.

Como exposto na metodologia, inicialmente analisamos as séries de internações e mortes sem

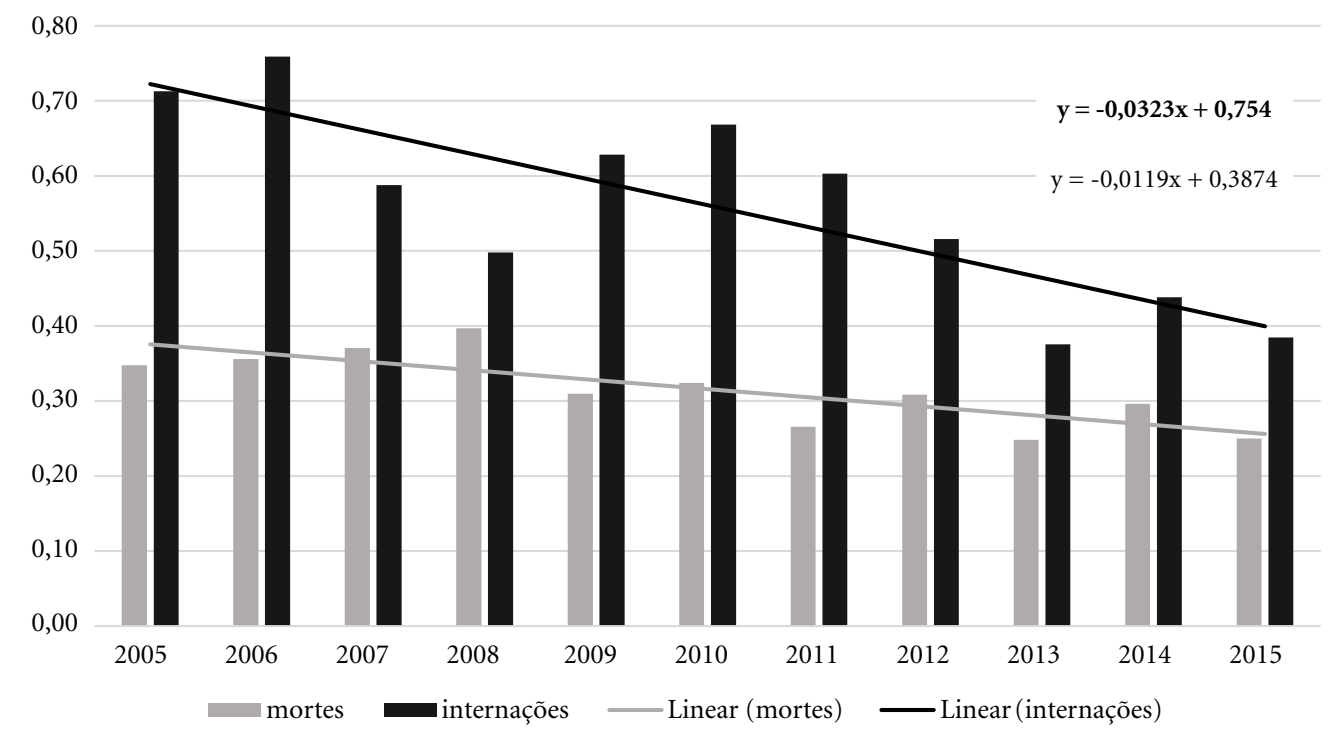

${ }^{1}$ CID 10: V40-V59; ${ }^{2}$ Automóveis, caminhonetas e caminhonetes.

Gráfico 1. Média mensal de internações e mortes de crianças de zero a quatro anos por acidentes de trânsito. 
a secção dos períodos entre "antes" e "depois" da obrigatoriedade dos dispositivos de retenção, ou seja, foram estudados os períodos completos, entre 2005 e 2015, das séries temporais de internações e mortes de crianças até quatros anos vítimas de sinistros de trânsito, utilizando a data de início da fiscalização como marco temporal para a análise de intervenção. A Tabela 1 descreve os resultados da análise de intervenção.

A Tabela 1 sugere, a partir dos resultados da variável dummy, que houve uma redução estatisticamente significativa, a um nível de confiança de 99\%, nas internações e nas mortes de crianças até quatro anos em decorrência de sinistros de trânsito após o período da obrigatoriedade da utilização dos dispositivos de retenção. Os resultados apontam para uma redução maior nas internações $(-0,127)$ do que nas mortes $(-0,074)$, corroborando com a sinalização apresentada no Gráfico 1. Dessa forma, os resultados da análise de intervenção foram compatíveis com a análise

Tabela 1. Resultados da análise das séries temporais de internações e mortes

\begin{tabular}{|c|c|c|c|}
\hline Variável & B & $\begin{array}{l}\text { Desvio } \\
\text { padrão }\end{array}$ & $\begin{array}{c}\text { Estatistica } \\
-t\end{array}$ \\
\hline \multicolumn{4}{|c|}{ Internações } \\
\hline $\mathrm{C}$ & $0.622^{* * *}$ & 0.029 & 2.123 .627 \\
\hline DUMMY & $-0.127^{\star * *}$ & 0.039 & -3.199 .784 \\
\hline $\mathrm{AR}(1)$ & $0.388^{\star * *}$ & 0.088 & 4.416 .895 \\
\hline SIGMASQ & $0.021^{* * *}$ & 0.003 & 6.785 .071 \\
\hline $\mathrm{R}^{2}$ & 0.291 & & \\
\hline $\mathrm{F}$ & 1.587 .854 & & \\
\hline Durbin-Watson & 2.127 & & \\
\hline \multicolumn{4}{|c|}{ Mortes } \\
\hline $\mathrm{C}$ & $0.350^{* * *}$ & 0.010 & 3.305 .813 \\
\hline DUMMY & $-0.074^{* * *}$ & 0.016 & -4.595 .320 \\
\hline $\mathrm{AR}(1)$ & $0.582^{\star * *}$ & 0.163 & 3.557 .701 \\
\hline $\operatorname{AR}(2)$ & $0.421^{\star \star}$ & 0.206 & 2.043 .389 \\
\hline $\operatorname{AR}(3)$ & $-0.567^{\star * *}$ & 0.197 & -2.864 .839 \\
\hline $\operatorname{AR}(4)$ & $-0.228^{\star *}$ & 0.106 & -2.149 .098 \\
\hline $\mathrm{MA}(1)$ & $-0.564^{* * *}$ & 0.157 & -3.574 .104 \\
\hline $\mathrm{MA}(2)$ & $-0.439^{* *}$ & 0.183 & -2.395 .355 \\
\hline $\mathrm{MA}(3)$ & $0.721^{\star \star *}$ & 0.148 & 4.871 .197 \\
\hline SIGMASQ & $0.008^{* * *}$ & 0.001 & 6.756 .917 \\
\hline $\mathrm{R}^{2}$ & 0.144 & & \\
\hline $\mathrm{F}$ & 4.848 .804 & & \\
\hline Durbin-Watson & 1.913 & & \\
\hline
\end{tabular}

$* * * 99 \%$, ** $95 \%, * 90 \%$.

Fonte: Elaborado pelos autores. descritiva, reforçando a indicação de redução das internações e mortes após a obrigatoriedade dos dispositivos de retenção.

A secção das séries temporais procurou confirmar os resultados da análise de intervenção. As séries temporais das internações e das mortes foram divididas em duas cada uma: a série denominada "antes" correspondeu ao período anterior à obrigatoriedade dos dispositivos de retenção, ou seja, entre setembro de 2005 e agosto de 2010; e a série denominada "depois" correspondeu ao período posterior à obrigatoriedade dos dispositivos, isto é, entre setembro de 2010 e agosto de 2015. A análise das séries apresentou os resultados descritos na Tabela 2.

Os resultados da Tabela 2 sugerem que houve redução no intercepto, tanto entre as séries das internações "antes" e "depois" (de C $=0.634$ para $C=0.494)$, como para as séries das mortes "antes" e "depois" (de C $=0.349$ para $C=0.273$ ). De forma sintética, podemos dizer que a redução no intercepto pode ser entendido como uma redução no ponto de partida das séries temporais após a obrigatoriedade dos dispositivos de retenção. Esses resultados são compatíveis com os resultados da análise de intervenção, apontando para a redução de internações e mortes de crianças entre zero e quatro anos após a obrigatoriedade da utilização dos dispositivos de retenção. Também houve compatibilidade com os resultados da análise de intervenção no que se refere à indicação de redução maior entre as internações do que entre as mortes.

No que se refere à tendência, os resultados mais uma vez indicam que a obrigatoriedade do uso dos dispositivos de retenção foi eficiente, já que a análise das séries das internações "antes" e "depois" sugere que houve uma redução na tendência de alta (de $\operatorname{AR}(1)=0,885$ para $\operatorname{AR}(1)=$ 0,551). Sinteticamente, podemos dizer que a redução na tendência de alta na inclinação das séries temporais das internações após a obrigatoriedade dos dispositivos de retenção significa que a série temporal ficou mais suave ou mais horizontal. No mesmo sentido, apontamos na análise das séries das mortes "antes" e "depois" a elevação da inclinação da tendência de baixa (AR(1) $=-0.567$ para $\operatorname{AR}(1)=-0.924)$. De forma alegórica, novamente, podemos dizer que a elevação da inclinação da tendência de baixa nas séries temporais das mortes após a obrigatoriedade dos dispositivos de retenção significa que a queda ficou mais acentuada. Em resumo, esses resultados sinalizam a suavização da tendência de aumento das internações e o incremento da tendência de 
Tabela 2. Análise das séries temporais de internações e mortes “antes” e "depois".

\begin{tabular}{|c|c|c|c|c|c|}
\hline \multicolumn{3}{|c|}{ Antes } & \multicolumn{3}{|c|}{ Depois } \\
\hline \multicolumn{6}{|c|}{ Internações } \\
\hline Variável & B & Desvio padrão & Variável & B & Desvio padrão \\
\hline $\mathrm{C}$ & $0.634^{* * *}$ & 0.061 & $\mathrm{C}$ & $0.494^{\star * *}$ & 0.041 \\
\hline $\operatorname{AR}(1)$ & $0.885^{\star \star *}$ & 0.126 & $\operatorname{AR}(1)$ & $0.551^{\star * *}$ & 0.105 \\
\hline $\mathrm{MA}(1)$ & $-0.688^{\star * *}$ & 0.222 & & & \\
\hline SIGMASQ & $0.022^{\star * *}$ & 0.004 & SIGMASQ & $0.017^{\star * *}$ & 0.003 \\
\hline $\mathrm{R}^{2}$ & 0.169 & & $\mathrm{R}^{2}$ & 0.278 & \\
\hline $\mathrm{F}$ & 3.806 .686 & & $\mathrm{~F}$ & 1.100 .780 & \\
\hline Durbin-Watson & 2.015 & & Durbin-Watson & 2.061 & \\
\hline \multicolumn{6}{|c|}{ Mortes } \\
\hline Variável & B & Desvio padrão & Variável & B & Desvio padrão \\
\hline $\mathrm{C}$ & $0.349^{* * *}$ & 0.0161 & $\mathrm{C}$ & $0.273^{\star * *}$ & 0.012 \\
\hline $\operatorname{AR}(1)$ & $-0.567^{\star \star}$ & 0.239 & $\operatorname{AR}(1)$ & $-0.924^{\star * *}$ & 0.156 \\
\hline \multirow[t]{3}{*}{$\mathrm{MA}(1)$} & $0.841^{\star * *}$ & 0.162 & $\operatorname{AR}(2)$ & 0.094 & 0.215 \\
\hline & & & $\operatorname{AR}(3)$ & $0.265^{\star}$ & 0.147 \\
\hline & & & $\mathrm{MA}(1)$ & $0.934^{\star * *}$ & 0.092 \\
\hline SIGMASQ & $0.009^{* * *}$ & 0.002 & SIGMASQ & $0.006^{\star * *}$ & 0.001 \\
\hline $\mathrm{R}^{2}$ & 0.098 & & $\mathrm{R}^{2}$ & 0.128 & \\
\hline $\mathrm{F}$ & 2.032 .724 & & $\mathrm{~F}$ & 1.585 .707 & \\
\hline Durbin-Watson & 1.969 & & Durbin-Watson & 1.935 & \\
\hline
\end{tabular}

Fonte: Elaborado pelos autores.

redução das mortes de crianças de zero a quatro anos em decorrência de sinistros de trânsito após a obrigatoriedade dos dispositivos de retenção, $\mathrm{o}$ que aponta que houve redução nas internações e mortes após a obrigatoriedade.

\section{Considerações finais}

A partir da realização da pesquisa, observamos a aderência dos resultados encontrados nas diferentes metodologias aplicadas, o que sugere que a exigência da utilização de dispositivos de retenção para o transporte de crianças de zero a quatro anos em veículos automotores trouxe resultados positivos para a redução de internações e mortes desse grupo em sinistros de trânsito no Brasil. Dessa forma, tal como sugere a Organização Mundial de Saúde ${ }^{1}$, há uma indicação de que a política de exigir a utilização de tais dispositivos para crianças de até quatro anos de idade seja eficiente para a redução de danos advindos de sinistros de trânsito.

Os resultados encontrados nesta pesquisa, apesar de obtidos por meio de uma metodologia distinta, foram compatíveis com os de outras pesquisas realizadas para analisar a relação entre o uso dos dispositivos de retenção e a redução de lesões e mortes de crianças em sinistros de trânsito. Em todos os casos, há a sugestão de que é mais seguro que as crianças sejam transportadas em dispositivos de retenção nos veículos automotores $^{10,12,13,22}$.

É digno de registro que o presente estudo primou pela robustez metodológica, isolando apenas os veículos que obrigatoriamente precisam transportar crianças nos dispositivos de retenção e contabilizando somente as internações e mortes de crianças pertencentes ao espectro da pesquisa. No entanto, não se pode ignorar a existência de outras variáveis que possam influir nas internações e mortes de crianças no trânsito e que não foram consideradas neste trabalho. Apontamos a falta de significância estatística para alguns elementos do modelo adotado para as séries das mortes depois da obrigatoriedade das cadeirinhas como uma limitação do estudo. Como sugestão para estudos futuros, sugerimos a aplicação regionalizada da metodologia aplicada na pesquisa, pois os dados nacionalizados utilizados não permitem a análise da relação entre a obrigatoriedade dos dispositivos de retenção e o número de internações e mortes de crianças nos diferentes estados da federação, por exemplo. 


\section{Colaboradores}

JCM Oliveira trabalhou na concepção, pesquisa e redação do artigo. LH Silva Júnior trabalhou na concepção e redação final do artigo. AN Almeida trabalhou na revisão dos resultados e na redação final do artigo.

\section{Referências}

1. World Health Organization (WHO). Global status report on road safety 2018. Geneva: WHO; 2018.

2. Instituto de Pesquisa Econômica Aplicada (Ipea). Estimativa dos custos dos sinistros de trânsito no Brasil com base na atualização simplificada das pesquisas anteriores do Ipea. Brasília: Ipea; 2015.

3. Bacchieri G, Barros AJ. Sinistros de trânsito no Brasil de 1998 a 2010: muitas mudanças e poucos resultados. Rev Saude Publica 2011; 45(5):949-63.

4. Guerreiro JVR, Campos CI, Lollo JA. Mapeamento espaço-temporal de fatalidades no trânsito brasileiro de crianças de 0-14 anos. In: XVI Congreso Chileno de Ingeniería de Transporte; 2013 Oct 21-25; Santiago, Chile. p. 1-13.

5. Souza MFM, Malta DC, Conceição GMS, Silva MMA, Gazal-Carvalho C, Morais Neto OL. Análise descritiva e de tendência de sinistros de transporte terrestre para políticas sociais no Brasil. Epidemiol Serv Saude 2007; 16(1):33-44.

6. Oliveira SRL, Leone C, Carvalho MDB, Santana RG, Lüders LE, Oliveira FC. Erros de utilização de assentos de segurança infantil por menores de 4 anos. J Pediatr (Rio J) 2012; 1(1):297-302.

7. Durbin DR, Hoffman BD. Child passenger safety. Pediatrics 2018; 142(5):1-8.

8. Scherz RG. Restraint systems for the prevention of injury to children in automobile accidents. Am J Public Health 1976; 66(5):451-456.

9. Elliott MR, Kallan MJ, Durbin DR, Winston FK. Effectiveness of child safety seats vs seat belts in reducing risk for death in children in passenger vehicle crashes. Arch Pediatr Adolesc Med 2006;160(6):617621.

10. Rice TM, Anderson CL. The effectiveness of child restraint systems for children aged 3 years or younger during motor vehicle collisions: 1996 to 2005. Am J Public Health 2009; 99(2):252-257.

11. Arbogast KB, Durbin DR, Cornejo RA, Kallan MJ, Winston FK. An evaluation of the effectiveness of forward facing child restraint systems. Accid Anal Prev 2004; 36(4):585-589.

12. Ma X, Layde P, Zhu S. Association between child restraint systems use and injury in motor vehicle crashes. Acad Emerg Med 2012; 19(8):916-923.

13. Zaloshinja E, Miller TR, Hendrie D. Effectiveness of child safety seats vs safety belts for children aged 2 to 3 years. Arch Pediatr Adolesc Med 2007; 161(1):65-68

14. Zaza S, Sleet DA, Thompson RS, Sosin DM, Bolen JC. Reviews of evidence regarding interventions to increase use of child safety seats. Am J Prev Med 2001; 21(Suppl. 4):31-47.

15. Kallan MJ, Durbin DR, Arbogast KB. Seating patterns and corresponding risk of injury among 0 - to 3-year-old children in child safety seats. Pediatrics 2008 ; 121(5): 342-1347.

16. Sauber-Schatz EK, West BA, Bergen G. Vital signs: restraint use and motor vehicle occupant death rates among children aged 0-12 years. MMWR Morb Mortal Wkly Rep 2014; 63(5):113-118.

17. Durbin DR. Technical report - child passenger safety. Pediatrics 2011; 121(4):1050-1066.

18. Brasil. Lei no 9.503 , de 23 de setembro de 1997. Institui o Código de Trânsito Brasileiro. Diário Oficial da União 1997; 24 set. 
19. Brasil. Conselho Nacional de Trânsito. Resolução no 277, de 28 de maio de 2008. Dispõe sobre o transporte de menores de 10 anos e a utilização do dispositivo de retenção para o transporte de crianças em veículos. Diário Oficial da União 2008; 19 jun.

20. Brasil. Departamento Nacional de Trânsito. Relatórios estatísticos [página na Internet]. [acessado 2017 Out 10]. Disponível em: http://www.denatran.gov.br/estatistica/237-frota-veiculos.

21. Sousa RM, Felisbino Júnior F, Braga FM, Costa Neto SD, Belo FM, Silva RS, Moraes FB. Uso correto do cinto de segurança e dos dispositivos de restrição infantil em automóveis de Goiânia. Rev Bras Ortop 2014; 49(4):340-344.

22. Silva M, Oyama SMR, Sanchez FFS. Dispositivos de segurança para crianças em veículos motorizados: prevenção de morbi-mortalidade. Perspectivas Médicas 2016; 27(2):9-15.

23. Campos CI, Raia Júnior AA. Políticas públicas de segurança viária para o transporte de crianças: o caso do Brasil. In: XIII Safety, Health and Environment World Congress; 2013; Porto, Portugal. p. 245-249.

24. Polli JB, Polli I. Traveling with children: beyond car seat safety. J Pediatr (Rio J) 2015; 91(6):515-522.

25. Brasil. Ministério da Saúde (MS). Datasus. Sistema de Informações Hospitalares. Internações por causas externas - Brasil - 2017 [página na Internet]. [acessado 2019 nov 3]. Disponível em: http://www2.datasus. gov.br/DATASUS/index.php?area $=0203 \& \mathrm{id}=6926 \&$ $\mathrm{VObj}=\mathrm{http}: / /$ tabnet.datasus.gov.br/cgi/deftohtm. exe?sih/cnv/ni.
26. Brasil. Ministério da Saúde (MS). Datasus. Sistema de Informações sobre Morbidades. Mortes por causas externas - Brasil - 2017 [página na Internet]. [acessado 2019 nov 3]. Disponível em: http://tabnet.datasus. gov.br/CGI/TABCGI.EXE?SIM/CNV/EXT10UF.DEF.

27. Mello Jorge MHP. Sinistros de trânsito no Brasil: um atlas de sua distribuição. São Paulo: ABRAMET; 2013.

28. Brasil. Conselho Nacional de Trânsito. Resolução no 352 , de 14 de junho de 2010. Dispõe sobre o transporte de menores de 10 anos e a utilização do dispositivo de retenção para o transporte de crianças em veículos. Diário Oficial da União 2010; 18 de jun.

29. Box GEP, Jenkins GM. Time series analysis: forecasting and control. London: Holden-Day; 1970.

30. Morettin PA, Toloi CMC. Análise de séries temporais. São Paulo: Blucher; 2006.

Artigo apresentado em 20/07/2018

Aprovado em 06/01/2020

Versão final apresentada em 08/01/2020

Editores-chefes: Romeu Gomes, Antônio Augusto Moura da Silva 